\title{
Cyclotron resonance in Kondo insulator
}

\author{
Yasuhiro Tada ${ }^{*}{ }^{*}$ \\ Institute for Solid State Physics, University of Tokyo, Kashiwa 277-8581, Japan
}

(Received 18 December 2019; accepted 28 April 2020; published 19 May 2020)

\begin{abstract}
Motivated by the recent experimental observations of quantum oscillations in the Kondo insulators $\mathrm{SmB}_{6}$ and $\mathrm{YbB}_{12}$, we study magneto-optical conductivity of Kondo insulators. We show that there exist nontrivial cyclotron resonance peaks in a frequency domain below the hybridization gap as a result of the characteristic Landau level structure of the system. Furthermore, these low-energy peaks are enhanced near the insulator-metal phase transition driven by an applied magnetic field and stable even at low temperatures. They are smoothly connected via the Kondo effect to resonance peaks for unhybridized conduction and $f$ electrons at high temperature. The nontrivial cyclotron resonance is a dynamical counterpart of quantum oscillations in static quantities, and through this intimate connection it will provide a useful insight for the latter.
\end{abstract}

DOI: 10.1103/PhysRevResearch.2.023194

\section{INTRODUCTION}

Metals and insulators are distinguished by their low-energy behaviors which are normally well characterized by the existence or absence of a Fermi surface. In particular, their difference becomes significant under a magnetic field, where the Fermi surface is discretized into Landau levels and quantum oscillations can be seen as a direct consequence in metals, while such behaviors are absent in insulators according to the standard theories [1]. However, these canonical understandings of metals and insulators are challenged by the recent experimental observations of quantum oscillations in the Kondo insulators $\mathrm{SmB}_{6}$ and $\mathrm{YbB}_{12}$ [2-5]. Quantum oscillations are observed in the magnetization of $\mathrm{SmB}_{6}$ [2,3], and the system becomes metallic for very strong magnetic fields, $B \gtrsim 100 \mathrm{~T}$ [6-9]. In $\mathrm{YbB}_{12}$, quantum oscillations are found when $B \sim$ $40 \mathrm{~T}[4,5]$, and a field-induced metallic phase is realized for $B \gtrsim 50 \mathrm{~T}$ [10-12]. In this way, both of these materials show quantum oscillations inside the insulating phases and they are enhanced as the system approaches the insulator-metal phase transition point. In addition, $\mathrm{SmB}_{6}$ and $\mathrm{YbB}_{12}$ are candidates of topological Kondo insulators $[13,14]$, and the possible relations of their topological nature to the quantum oscillations attract great attention.

To understand these anomalous quantum oscillations, a number of theories have been proposed [15-24]. Although theoretical understandings have not yet been settled, it was demonstrated that quantum oscillations can be found not only in Kondo insulators, but also in general insulators with band inversion because Landau levels change their character between a conduction-electron-like one and $f$-electron-like

\footnotetext{
*tada@issp.u-tokyo.ac.jp

Published by the American Physical Society under the terms of the Creative Commons Attribution 4.0 International license. Further distribution of this work must maintain attribution to the author(s) and the published article's title, journal citation, and DOI.
}

one successively as the magnetic field increases [15-21]. This is a hallmark of the characteristic Landau level structure realized in a Kondo insulator, which is totally different from that in a conventional semiconductor or a simple insulator without band inversion. Thus far, unusual Landau levels and anomalous properties under magnetic fields have been extensively studied in exotic semimetals such as graphene and topological materials [25-27]. Now, band inverted insulators provide a new platform for physics under strong magnetic fields. In order to pin down the origin of the observed quantum oscillations and elucidate the impacts of magnetic fields, it is desirable to clarify the Landau level structures and their physical consequences in detail. However, the previous theoretical studies focus only on static quantities, while a dynamical quantity may provide rich information for clarification of the unusual Landau level structures and also for a possible origin of the observed quantum oscillations.

In this study, we investigate cyclotron resonance in Kondo insulators. The cyclotron resonance can directly access a Landau level structure by the frequency dependence of the magneto-optical conductivity, where a resonance peak corresponds to a transition between different Landau levels. Historically, it was first developed mainly for semiconductors and later employed for studying semimetallic graphene with unusual Landau levels [25,28-32]. Recently, it was also applied to metals with strong correlations such as the candidate topological superconductor $\mathrm{Sr}_{2} \mathrm{RuO}_{4}$, Fe-based superconductor $\mathrm{KFe}_{2} \mathrm{As}_{2}$, and hidden-order phase of the $f$-electron material $\mathrm{URu}_{2} \mathrm{Si}_{2}$ [33-35]. Similarly to the quantum oscillations in static quantities, it has been naively expected that low-energy cyclotron resonance can be obtained only for a system with conducting carriers, while a resonance peak could be seen only for an energy scale above a gap in insulators. Here, we demonstrate that there exist nontrivial cyclotron resonance peaks well below an activation gap in a Kondo insulator at low temperature. Furthermore, the low-energy resonance peaks are enhanced near the insulator-metal quantum phase transition point. These anomalous behaviors are a direct consequence of the characteristic Landau level structures which are 
also responsible for quantum oscillations. This is a dynamical counterpart of quantum oscillations in static quantities, and through this intimate connection it will provide a clue for the mechanism of the latter.

\section{MODEL AND CALCULATIONS}

\section{A. Hamiltonian and its spectrum}

We consider a simple model of a Kondo insulator composed of single orbital conduction electrons and $f$ electrons with pseudospins in two dimensions [15-24]. The effects of three dimensionality will be discussed later. Since our primary purpose is to clarify the cyclotron resonance in a general Kondo insulator, we consider both a nontopological insulator and topological insulator on an equal footing. The Hamiltonian is $H=\int d^{2} x \psi^{\dagger}(\boldsymbol{x}) \mathcal{H} \psi(\boldsymbol{x})$,

$$
\mathcal{H}=\left(\begin{array}{cc}
\frac{\pi^{2}}{2 m_{c}}+\frac{g_{c} \mu_{B}}{2} \sigma_{z} B & v_{0}+v_{1} a \boldsymbol{\pi} \cdot \boldsymbol{\sigma}^{t} \\
v_{0}+v_{1} a \boldsymbol{\pi} \cdot \boldsymbol{\sigma}^{t} & -\frac{\pi^{2}}{2 m_{f}}+\epsilon_{f 0}+\frac{g_{f} \mu_{B}}{2} \sigma_{z} B
\end{array}\right),
$$

where $\psi(\boldsymbol{x})=\left(\psi_{c \uparrow}, \psi_{c \downarrow}, \psi_{f \uparrow}, \psi_{f \downarrow}\right)$ is the field operator for the conduction and $f$ electrons. $\pi=-i \hbar \nabla+e \boldsymbol{A}$ with the electric charge $-e<0$ is the dynamical momentum and $\boldsymbol{A}$ is a vector potential for a uniform magnetic field $\boldsymbol{B}=\boldsymbol{\nabla} \times$ $\boldsymbol{A}=(0,0, B)$ with $B>0 . m_{c}, m_{f}$ are effective masses for the conduction electrons and $f$ electrons respectively, and $m_{c}$ is assumed to equal the bare electron mass $m_{0}$ and $m_{f}=0.1 m_{c}$ for brevity. $\epsilon_{f 0}$ is the $f$-electron energy level, and $v_{0}, v_{1}$ represent hybridization strength with a characteristic lattice length scale $a \sim$ few $\AA$, which is taken to be the length unit. The system is a topological Kondo insulator when $v_{0}=$ $0, v_{1} \neq 0$, while it is a nontopological one for $v_{0} \neq 0, v_{1}=0$ $[13,14] . \mu_{B}=e \hbar / 2 m_{0}$ is the Bohr magneton, and $g$ factors are assumed to be $g_{c}=g_{f}=2$ for simplicity. We use the energy unit $t_{0} \equiv \hbar^{2} /\left(2 m_{0} a^{2}\right)$, and set $\hbar=1$ and the Boltzmann constant $k_{B}=1$. We fix the model parameters as $\epsilon_{f 0}=0.5 t_{0}$, and $\left(v_{0}, v_{1}\right)=\left(0.05 t_{0}, 0\right)$ for a nontopological insulator and $\left(v_{0}, v_{1}\right)=\left(0,0.05 t_{0}\right)$ for a topological insulator, where the activation gaps at zero magnetic field are $\Delta_{0}=2 v_{0}=0.1 t_{0}$ and $\Delta_{1}=\sqrt{\epsilon_{f 0}^{2}-\left[4\left(v_{1} a\right)^{2} m_{c} m_{f} /\left(m_{c}+m_{f}\right)\right]^{2}} \simeq 0.067 t_{0}$, respectively. Although these parameters may be too large when considering the Kondo insulators $\mathrm{SmB}_{6}$ and $\mathrm{YbB}_{12}$, they will be renormalized by the strong Coulomb interactions between the $f$ electrons as $m_{f} \rightarrow m_{f} / z, v_{0,1} \rightarrow \sqrt{z} v_{0,1}$ with a renormalization factor $z \ll 1$. We have confirmed that our results are qualitatively unchanged when we use different parameter values.

It is straightforward to diagonalize the Hamiltonian (1). We expand the field operator as $\psi_{i}(\boldsymbol{x})=\sum_{n k} a_{n k i} \phi_{n k}(\boldsymbol{x})$ [ $i$ represents $i=(c, \uparrow)$, etc.] by using the harmonic oscillator single-particle wave functions $\phi_{n k}$ with a Landau level $n$ and an index for degeneracy $k . a_{n k}$ is the corresponding annihilation operator. For a system in the infinite cylinder geometry with the size $L_{x}=\infty, L_{y}<\infty$, the lowest Landau level wave function is $\phi_{0 k}=1 / \sqrt{\sqrt{\pi} l_{B} L_{y}} \exp [i k y-(x-$ $\left.k l_{B}^{2}\right)^{2} / 2 l_{B}^{2}$ ] under the Landau gauge $\boldsymbol{A}=(0, B x, 0)$. They satisfy $l_{B} \pi_{+} \phi_{n k}=\sqrt{n+1} \phi_{n+1 k}, l_{B} \pi_{-} \phi_{n k}=\sqrt{n} \phi_{n-1 k}$, where $\pi_{ \pm}=\left(\pi_{x} \pm i \pi_{y}\right) / \sqrt{2}$ and $l_{B}=1 / \sqrt{e B}$ is the magnetic length.
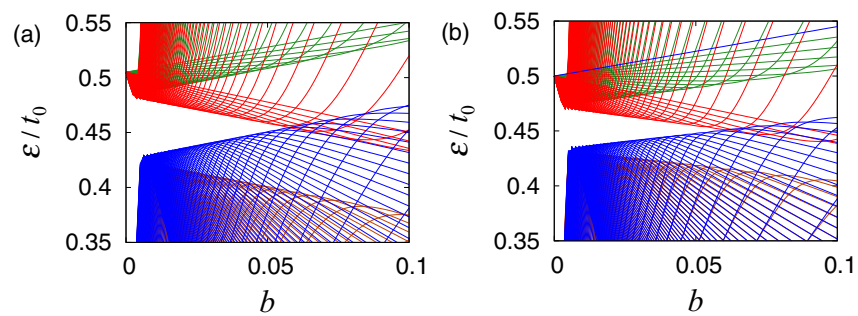

FIG. 1. Energy spectra of (a) the nontopological and (b) the topological Kondo insulators. The dimensionless magnetic field is $b=e B /\left(m_{0} t_{0}\right)=4 \pi B a^{2} / \phi_{0}$. The different colors correspond to the four eigenvalues $\left\{\varepsilon_{n m}\right\}_{m=1}^{4}$ for each Landau level $n$.

We suppress the index $k$ because it is not directly involved in the following discussions. The Hamiltonian $\mathcal{H}_{n, i j}$ in the Landau level basis gives eigenvalues $\left\{\varepsilon_{n m}\right\}_{m=1}^{4}$ and eigenvectors $\left\{u_{n, i m}\right\}_{m=1}^{4}[15,16]$. The energy spectra for both the nontopological and topological cases are shown in Fig. 1. The gap closes at a critical value $b_{c} \simeq 0.058$ with $b \equiv e B /\left(m_{0} t_{0}\right)=$ $4 \pi B a^{2} / \phi_{0}\left(\phi_{0}=h / e\right.$ is the magnetic flux quantum) for both $\left(v_{0}, v_{1}\right)=\left(0.05 t_{0}, 0\right),\left(v_{0}, v_{1}\right)=\left(0,0.05 t_{0}\right)$ and the system becomes metallic for $b>b_{c}$, corresponding to a field-induced metallic phase as seen in $\mathrm{SmB}_{6}$ and $\mathrm{YbB}_{12}$ [6-12]. For simplicity, we set the chemical potential so that it coincides with the energy where the gap closes, $\mu=0.454 t_{0}$ for the nontopological case and $\mu=0.45 t_{0}$ for the topological case, respectively. The Landau level structure is complicated, but, roughly speaking, it is a combination of $\varepsilon_{c n}=\omega_{c}(n+1 / 2)$ and $\varepsilon_{f n}=-\omega_{f}(n+1 / 2)+\epsilon_{f 0}$ with Zeeman shifts, and Landau levels change their characters between the conductionelectron-like one and $f$-electron-like one successively as $b$ increases. Because of the hybridization $v_{0,1}$, energy spacing between the neighboring Landau levels is not exactly $\omega_{c}, \omega_{f}$, but can be in between them especially when the crossover in the Landau level's character takes place, which is responsible for quantum oscillations in static quantities. We will see in the following that the magneto-optical conductivity can faithfully reflect these characteristic structures, providing rich information on the unusual Landau levels.

\section{B. Brief overview of dHvA effect}

Before going on to the discussion of cyclotron resonance, we first give a brief overview of the quantum oscillations of orbital magnetization at zero temperature [de Haas-van Alphen (dHvA) effect] to provide an intuitive insight of the characteristic Landau levels [15]. This will be helpful for understanding the intimate relationship between the quantum oscillations and cyclotron resonance discussed in the next section. In this section, we keep the Planck constant $\hbar$ explicitly. For simplicity, we ignore the Zeeman effect and consider $m_{f} \rightarrow \infty, v_{0} \neq 0, v_{1}=0$ (a similar discussion holds for the topological $v_{0}=0, v_{1} \neq 0$ case). In this case, the energy spectrum is given by

$$
\varepsilon_{n \pm}=\frac{1}{2}\left[\varepsilon_{c n}+\epsilon_{f 0} \pm \sqrt{\left(\varepsilon_{c n}-\epsilon_{f 0}\right)^{2}+4 v_{0}^{2}}\right],
$$

where $\varepsilon_{c n}=\hbar \omega_{c}(n+1 / 2)$. At the zero-temperature limit $T \rightarrow 0$, only the lower energies $\varepsilon_{n-}<\mu$ contribute to the ground-state energy. In the limit of small hybridization $v_{0} \ll$ 


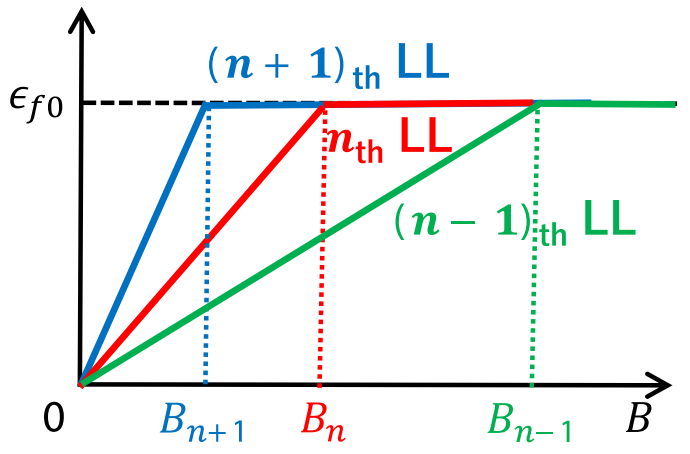

FIG. 2. The approximate Landau level (LL) spectrum $\left\{\varepsilon_{n-}\right\}$ [Eq. (3)] and the crossover fields $\left\{B_{n}\right\}$. Only three Landau levels are shown for simplicity.

$\epsilon_{f 0}$, it behaves as $\varepsilon_{n-} \simeq \varepsilon_{n c}-\left(\varepsilon_{c n}^{2}+4 v_{0}^{2}\right) / \epsilon_{f 0}$ for $B \ll B_{n}$, while $\varepsilon_{n-} \simeq \epsilon_{f 0}-\left(\epsilon_{f 0}^{2}+4 v_{0}^{2}\right) / \varepsilon_{c n}$ for $B \gg B_{n}$, where $B_{n}$ is the crossover scale between the conduction-electron-like character and $f$-electron-like character defined by $\hbar \omega_{c}(n+$ $1 / 2)=\epsilon_{f 0}$ or, equivalently, $B_{n}=m_{c} \epsilon_{f 0} /[e \hbar(n+1 / 2)]$. To perform a simple analytical calculation, we introduce an approximation where $\varepsilon_{n-}$ is replaced by

$$
\varepsilon_{n-}= \begin{cases}\varepsilon_{c n} & \left(B \leqslant B_{n}\right) \\ \epsilon_{f 0} & \left(B>B_{n}\right) .\end{cases}
$$

The schematic behavior of $\varepsilon_{n-}$ is shown in Fig. 2. When the magnetic field is $B_{n+1}<B<B_{n}$, the ground-state energy density is

$$
\begin{aligned}
u(B) & =D \sum_{l=0}^{n} \varepsilon_{l-}+\tilde{u} \\
& =D \frac{\hbar \omega_{c}}{2}[n(n+1)+n+1]+\tilde{u},
\end{aligned}
$$

where $D=2 /\left(2 \pi l_{B}^{2}\right)=e B / \pi \hbar$ is the degeneracy per area including spin degrees of freedom and $\tilde{u}=D \sum_{l>n} \varepsilon_{l-}=$ $D \sum_{l>n} \epsilon_{f 0}$ is the contribution from higher Landau levels $n+1, n+2, \ldots$, with an appropriate regularization to avoid unphysical divergence. Similarly, the ground-state energy density for $B_{n}<B<B_{n-1}$ is

$$
u(B)=D \frac{\hbar \omega_{c}}{2}[(n-1) n+n]+D \epsilon_{f 0}+\tilde{u},
$$

where the second term is the contribution from $\varepsilon_{n-}$ and the functional form of $\tilde{u}$ is the same as above. Correspondingly, the orbital magnetization $M=-\partial u / \partial B$ is given by

$$
\begin{aligned}
& M\left(B_{n+1}<B<B_{n}\right)=-\frac{e \omega_{c}}{\pi}[n(n+1)+n+1]+\tilde{M}, \\
& M\left(B_{n}<B<B_{n-1}\right)=-\frac{e \omega_{c}}{\pi}[(n-1) n+n]-\frac{e \epsilon_{f 0}}{\pi \hbar}+\tilde{M},
\end{aligned}
$$

where $\tilde{M}(B)=-\partial \tilde{u} / \partial B$ and does not have a singularity. From these expressions, we see that the magnetization jumps at $B=$ $B_{n}$ and oscillates with the period $\delta(1 / B)=1 / B_{n}-1 / B_{n-1}=$ $e \hbar /\left(m_{c} \epsilon_{f 0}\right)$, which is inversely proportional to the area of a Fermi-surface cross section without hybridization when $\epsilon_{f 0} \simeq \mu$. The size of the jump gives the oscillation amplitude, $\delta M\left(B=B_{n}\right)=M\left(B_{n}+0\right)-M\left(B_{n}-0\right)=e \epsilon_{f 0} /(\pi \hbar)$. Note that the sharp discontinuity of $M(B)$ is due to the approximation given by Eq. (3), where $\varepsilon_{n-}$ is not differentiable at $B=B_{n}$, and $M(B)$ is smooth for the original spectrum given by Eq. (2). Note, also, that the condition $m_{f} \rightarrow \infty$ can be relaxed and the quantum oscillation can be described by a similar argument for $m_{f}<\infty$.

From the above derivation of the oscillating magnetization $M(B)$ where all the states below the chemical potential contribute, it is clear that the crossover of a Landau level character as a function of $B$ from a conduction-electron-like one to an $f$ electron-like one is the most important key. This observation also holds true for the quantum oscillation for static transport (Shubnikov-de Haas effect) where an effective density of states thermally smeared around the chemical potential will play a central role [16]. The smeared density of states also oscillates due to the crossover of the Landau level character, as easily expected from Fig. 1 where a Landau level energy approaches the band edge and goes away from it as $B$ increases. Therefore, we conclude that the quantum oscillations in static quantities are essentially due to the Landau level crossover in the present model. Note that there are densely distributed Landau levels near the edges of the upper and lower bands in Fig. 1 because energy-level spacings becomes narrower when the slope of a Landau level energy changes around the crossover field strength. In the next section, we will see that this characteristic structure of the Landau levels leads to low-energy cyclotron resonance, sharing essentially the same origin with the quantum oscillations of static quantities.

\section{Cyclotron resonance}

We calculate the magneto-optical conductivity for a circularly polarized light with frequency $\omega$ [36],

$$
\begin{gathered}
\operatorname{Re} \sigma_{-+}(\omega)=\frac{1}{\omega}\left[\operatorname{Im} K_{-+}^{R}(\omega)-\operatorname{Im} K_{-+}^{R}(0)\right], \\
K_{-+}^{R}(\omega)=\frac{-i}{V} \int d t \theta(t)\left\langle\left[J_{-}(t), J_{+}(0)\right]\right\rangle e^{i \omega t} \\
=\sum_{n m m^{\prime}} M_{n, m m^{\prime}}^{-+} \chi_{n, m m^{\prime}}^{-+}(\omega), \\
\chi_{n, m m^{\prime}}^{-+}(\omega)=\frac{f\left(\varepsilon_{n+1, m}\right)-f\left(\varepsilon_{n m^{\prime}}\right)}{\omega-\varepsilon_{n+1, m}+\varepsilon_{n m^{\prime}}+i \gamma},
\end{gathered}
$$

where $V$ is the volume of the system and $f(\varepsilon)=$ $\left(e^{(\varepsilon-\mu) / T}+1\right)^{-1}$. Similarly, the conductivity for the opposite polarization $\sigma_{+-}(\omega)$ is given by the correlation function $K_{+-}^{R}(\omega)=\frac{-i}{V} \int d t \theta(t)\left\langle\left[J_{+}(t), J_{-}(0)\right]\right\rangle e^{i \omega t}$, and satisfies $\operatorname{Re} \sigma_{-+}(\omega)=\operatorname{Re} \sigma_{+-}(-\omega)$. The current operators are given by $J_{+}=J_{-}^{\dagger}=J_{x}+i J_{y}=\sum \mathcal{J}_{+n, i j} a_{n+1, i}^{\dagger} a_{n j}$ with $J_{x, y}=$ $\int d^{2} x \psi^{\dagger} \partial \mathcal{H} / \partial A_{x, y} \psi$, and $\mathcal{J}_{n+}=\mathcal{J}_{n-}^{\dagger}$ is defined by

$$
\mathcal{J}_{n+}=-\sqrt{2} e\left(\begin{array}{cccc}
\frac{\sqrt{n+1}}{m_{c} l_{B}} & 0 & 0 & \sqrt{2} v_{1} a \\
0 & \frac{\sqrt{n}}{m_{c} l_{B}} & 0 & 0 \\
0 & \sqrt{2} v_{1} a & -\frac{\sqrt{n+1}}{m_{f} l_{B}} & 0 \\
0 & 0 & 0 & -\frac{\sqrt{n}}{m_{f} l_{B}}
\end{array}\right) .
$$



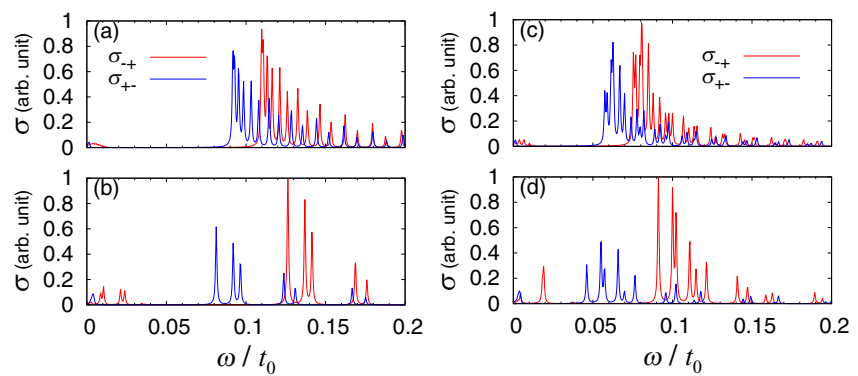

FIG. 3. Real part of the magneto-optical conductivity (in arbitrary units) for (a), (b) the nontopological insulator at $T=0.007 t_{0}=$ $0.07 \Delta_{0}$ and (c), (d) the topological insulator at $T=0.0047 t_{0} \simeq$ $0.07 \Delta_{1}$. The magnetic field is (a), (c) $b=0.02 \simeq 0.33 b_{c}$ and (b), (d) $b=0.05 \simeq 0.83 b_{c}$. The red curve is $\operatorname{Re} \sigma_{-+}(\omega)$ and the blue one is $\operatorname{Re} \sigma_{+-}(\omega)$.

The matrix $M$ is defined as $M_{n, m m^{\prime}}^{-+}=$ $\operatorname{tr}\left[\mathcal{J}_{n-} \mathcal{U}_{n+1 m} \mathcal{J}_{n+} \mathcal{U}_{n m^{\prime}}\right] /\left(2 \pi l_{B}^{2}\right)$ with $\left(\mathcal{U}_{n m}\right)_{i j}=u_{n, i m} u_{n, j m}^{*}$. We fix the phenomenological broadening parameter $\gamma=0.0005 t_{0}$ and the results are essentially independent of $\gamma$.

Figure 3 shows the magneto-optical conductivity $\sigma(\omega)$ at a low temperature $T=0.007 t_{0}=0.07 \Delta_{0}$ for the nontopological case and $T=0.0047 t_{0} \simeq 0.07 \Delta_{1}$ for the topological case. The calculated cyclotron resonance is qualitatively similar for these two cases, although there are some quantitative differences. For the small magnetic field $b=0.02 \simeq 0.33 b_{c}$, the conductivity is strongly suppressed and there are only small spectral weights in a low-energy region below the activation gap, $\omega<\Delta_{0,1}$. At high energy, a series of large peaks is located for $\omega \gtrsim \Delta_{0,1}$. On the other hand, when the magnetic field is close to the critical value, $b=0.05 \simeq 0.83 b_{c}, \sigma(\omega)$ at low energy is enhanced and there exist clear peaks well below the gap $\Delta_{0,1}$ even in the insulating states. More precisely, the low-energy peaks are located in the region $\omega_{f}=0.005 t_{0} \leqslant$ $\omega \leqslant \omega_{c}=0.05 t_{0}$ and will be called anomalous cyclotron resonance. The peak height is relatively larger in the topological case than in the nontopological case, and we conclude that the existence of the anomalous peaks is a universal property of Kondo insulators with band inversion. Furthermore, we will see later that the anomalous cyclotron resonance shares common features in both nontopological and topological cases. Such anomalous low-energy peaks are absent in non-bandinverted insulators, but are made possible because of the characteristic Landau level structures in the Kondo insulators. As seen in Fig. 1, there are Landau levels densely distributed near the edges of the lowest positive-energy band $\left(\varepsilon_{n m}>\mu\right.$, shown in red) and the highest negative-energy band $\left(\varepsilon_{n m}<\mu\right.$, shown in blue), corresponding to the previously mentioned crossover in the Landau level's character. Transitions between the $n \leftrightarrow n+1$ Landau levels within each band edge in the same color are allowed at finite temperature, resulting in the low-energy peaks in $\sigma(\omega)$, while such band edge Landau levels are absent in a non-band-inverted insulator. Therefore, these anomalous peaks are a direct consequence of the unusual Landau level structure, essentially sharing the common origin with quantum oscillations within the present model.
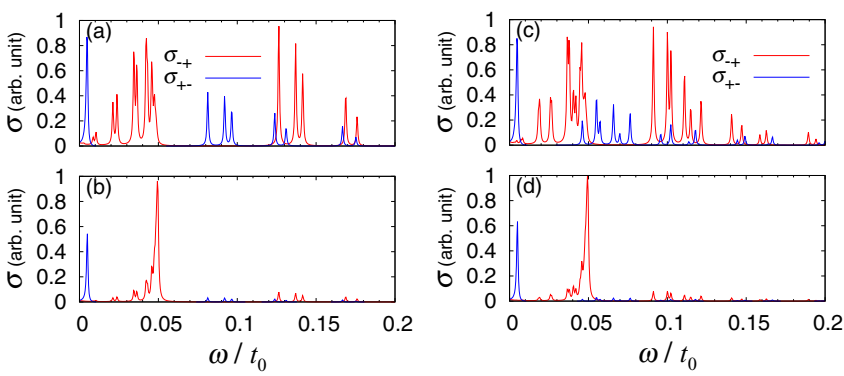

FIG. 4. Real part of the magneto-optical conductivity (in arbitrary units) for (a), (b) the nontopological insulator and (c), (d) the topological insulator at $b \simeq 0.83 b_{c}$. Temperature is (a) $T=0.05 t_{0}=$ $0.5 \Delta_{0}$, (b) $T=0.2 t_{0}=2 \Delta_{0}$, (c) $T=0.034 t_{0} \simeq 0.5 \Delta_{1}$, and (d) $T=$ $0.13 t_{0} \simeq 2 \Delta_{1}$. The red curve is $\operatorname{Re} \sigma_{-+}(\omega)$ and the blue one is $\operatorname{Re} \sigma_{+-}(\omega)$.

An observation of these peaks will strongly support the mechanism for quantum oscillations proposed in the previous theories [15-21], and hence provide a clue for the origin of the experimentally observed quantum oscillations. If the magnetic field is increased further, $b>b_{c}$, so that the system is metallic, one can see that the low-energy peaks are further enhanced, corresponding to heavy fermion formation in the metallic regime. For the Kondo insulators $\mathrm{SmB}_{6}$ and $\mathrm{YbB}_{12}$, the frequency of the light should be smaller than $\sim 1-2 \mathrm{meV}$ corresponding to their insulating gaps, and also in between $\omega_{f}$ and $\omega_{c}$ (they are $\omega_{f} \sim 0.1 \mathrm{meV}, \omega_{c} \sim 1 \mathrm{meV}$, for example, at $B=10 \mathrm{~T}$ in the present model). Besides, a large magnetic field $B>10 \mathrm{~T}$ would be required to observe the anomalous cyclotron resonance.

Next, we discuss the temperature dependence of the conductivity. Figure 4 shows $\sigma(\omega)$ for different temperatures at the magnetic field $b=0.05 \simeq 0.83 b_{c}$. At an intermediate temperature $T=0.5 \Delta_{0,1}$, the anomalous low-energy peaks are now comparable to the high-energy peaks. Similarly to the $T=0.07 \Delta_{0,1}$ case, these low-energy peaks in $\sigma_{-+}(\omega)$ are located in the region $\omega_{f}=0.005 t_{0} \leqslant \omega \leqslant \omega_{c}=0.05 t_{0}$, while there is only one low-energy peak at $\omega \simeq \omega_{f}$ in $\sigma_{+-}(\omega)$. The low-energy peaks become sharper at high temperature, $T=2 \Delta_{0,1}$, and there is basically a single peak at $\omega=\omega_{c}$ in $\sigma_{-+}(\omega)$ and at $\omega=\omega_{f}$ in $\sigma_{-+}(\omega)$. These two peaks at high temperature simply correspond to the unhybridized conduction electrons and $f$ electrons, which are directly seen in Eq. (8); contributions from high-energy states with large Landau level indices $n$ become dominant at very high temperature since their momenta $\pi_{ \pm} \sim \sqrt{n} / l_{B}$ are large, and hence one can neglect hybridization matrix elements $v_{0}, v_{1} a \sqrt{n} / l_{B} \ll$ $\omega_{c} n, \omega_{f} n$ in the Hamiltonian $\mathcal{H}_{n}$. Then a straightforward calculation leads to $\sigma_{-+}(\omega \geqslant 0) \propto \omega_{c} /\left(m_{c}^{2} T\right) \delta\left(\omega-\omega_{c}\right)$ and $\sigma_{+-}(\omega \geqslant 0) \propto \omega_{f} /\left(m_{f}^{2} T\right) \delta\left(\omega-\omega_{f}\right)$ in the limit $\gamma \rightarrow 0$. The conduction and $f$ electrons form a hybridized state at low temperature for which energy levels are nonlinear in the magnetic field $B$ and located in between the unhybridized conduction-electron energy and $f$-electron energy. This evolution of the system is a (noninteracting variant of the) Kondo effect and would be useful for studying the evolution of temperature-dependent electronic structures under magnetic 

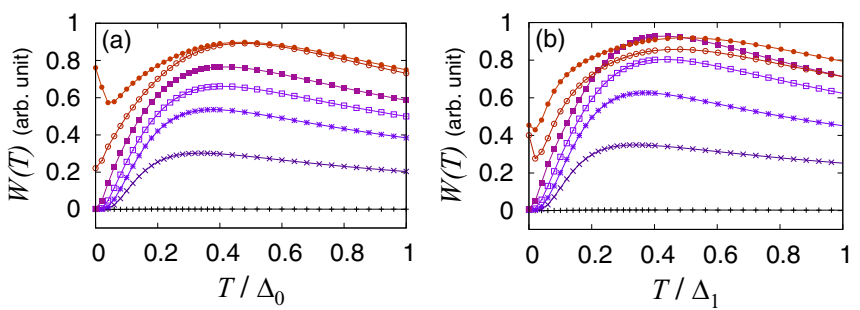

FIG. 5. Temperature dependence of the low-energy spectral weight $W_{-+}$in (a) the nontopological and (b) the topological Kondo insulators. The magnetic fields are $b=0.01,0.02,0.03$, $0.04,0.05,0.06,0.07$ from the bottom (black) to top (dark orange). $W_{-+}$for $b=0.01$ is much smaller than those for $b \geqslant 0.02$.

fields where photoemission experiments [37,38] are generally difficult.

The temperature dependence is well characterized by lowenergy spectral weight of the conductivity,

$$
W_{-+}=\int_{\Omega_{1}}^{\Omega_{2}} d \omega \operatorname{Re} \sigma_{-+}(\omega) .
$$

We choose the cutoff energy scales $\Omega_{1,2}$ so that only the anomalous low-energy peaks are taken into account; $\Omega_{1}=0$ and $\Omega_{2}=\omega_{c}-10 \gamma$ for a given magnetic field. Although the precise value of $W_{-+}$depends on a choice of $\Omega_{1,2}$, contributions from unhybridized conduction and $f$ electrons are suppressed with the present $\Omega_{1,2}$. In addition, only the transitions between states at Landau levels $n \leftrightarrow n+1$ with energies $\Omega_{1} \lesssim\left|\varepsilon_{n+1, m}-\varepsilon_{n m^{\prime}} \pm \gamma\right| \lesssim \Omega_{2}$ contribute to $W_{-+}$. Figure 5 shows $W_{-+}$for several values of magnetic fields $b$. We see that $W_{-+}$develops as the temperature is reduced via the Kondo effect and it becomes maximum roughly around $T \sim$ $0.3 \Delta_{0,1}-0.4 \Delta_{0,1}$. At low temperature, the weight behaves as $W_{-+}(T) \sim \exp \left(-\Delta_{-+} / T\right)$ with a gap $\Delta_{-+}$in the insulating regime. $\Delta_{-+}$corresponds to the minimum value of $\left|\varepsilon_{n m}-\mu\right|$ for the given magnetic field because the temperature factor of $W_{-+}$behaves as $\left|f\left(\varepsilon_{n+1, m}\right)-f\left(\varepsilon_{n m^{\prime}}\right)\right| \simeq e^{-\left|\varepsilon_{n+1, m}-\mu\right| / T}(1-$ $e^{-\left|\varepsilon_{n+1, m}-\varepsilon_{n m^{\prime}}\right| / T}$ ), e.g., for $\left|\varepsilon_{n+1, m}-\mu\right|<\left|\varepsilon_{n m^{\prime}}-\mu\right|$. $W_{-+}$is enhanced as the magnetic field increases in both nontopological and topological Kondo insulators in a similar fashion, which again means that the enhancement is a universal property of the Kondo insulators with band inversion. In this way, the low-energy peaks can become pronounced even in the insulating regime when the system is close to the insulator-metal transition point. The gap $\Delta_{-+}$becomes zero at the critical point $b=b_{c} \simeq 0.058$. For larger magnetic fields $b=0.06,0.07>b_{c}$ where the system is metallic, there exist resonance peaks in $\sigma_{-+}(\omega)$ in between $\omega_{f} \leqslant \omega \leqslant \omega_{c}$ corresponding to heavy fermions and $W_{-+}$remains nonzero at $T=0$.

Our results for the clean noninteracting model imply that the anomalous low-energy peaks corresponding to the characteristic Landau level structure are most clearly visible around an intermediate-temperature regime $\left(T \sim 0.3 \Delta_{0,1}-0.4 \Delta_{0,1}\right.$ in the above calculation). In real materials, however, sharp peaks will be smeared by disorder and interactions, especially at temperatures above the Kondo temperature which is roughly $T_{K} \sim \Delta_{0,1}$ [39-43], and sufficiently low temperature would be required for an experiment. At the same time, signal amplitudes would be reduced at low temperature, which is a common subtle issue also for observables where only states near the chemical potential contribute $[17,44]$. Nevertheless, quantum oscillations in static longitudinal transport have indeed been observed not only in $\mathrm{YbB}_{12}$, but also in band-inverted insulating quantum wells at low temperature $[4,45,46]$, and discussed theoretically [21]. Since cyclotron resonance can be observed at relatively high temperature in general, anomalous cyclotron resonance can be seen in some intermediate-temperature range below the Kondo temperature and provide a useful insight for the observed quantum oscillations.

Finally, we qualitatively discuss the effects of three dimensionality for which the response function is $\sigma\left(\omega, q_{z}\right)$ for a light perpendicular to the $x y$ plane with an in-plane polarization. Since we are interested in the frequency range below the activation gap $\Delta_{0,1}=O(\mathrm{meV})$ or even smaller due to the renormalization effect, the wavelength of the light used in a cyclotron resonance experiment will be much longer than a quasiparticle mean free path. Such a long wavelength may be regarded as the $q_{z} \rightarrow 0$ limit and a resonance peak position at $\omega=\left[\varepsilon_{n+1, m}\left(k_{z}+q_{z}\right)-\varepsilon_{n m^{\prime}}\left(k_{z}\right)\right]$ remains almost unchanged from that for a two-dimensional system. Therefore, the qualitative behaviors discussed in the present study will still hold for three dimensions. In case of a topological Kondo insulator, there are Zeeman split surface states and they may give additional contributions to cyclotron resonance, but they would be subdominant for a thick sample. In a thin film, however, the surface-mode contribution could be significant. A cyclotron resonance experiment with tilted field angles may provide useful information about the dimensionality of the carriers for the quantum oscillations. There is another additional contribution even in two dimensions arising from electron spins, but it could be separated from cyclotron resonance because its magnitude is usually small and the characteristic energy scale is Zeeman energy, which differs from a cyclotron frequency especially in an $f$-electron system. The spin response under a magnetic field itself will contain important information on electronic structures similarly to those at zero magnetic field $[47,48]$.

\section{SUMMARY}

We have investigated cyclotron resonance in Kondo insulators. We found that there exist anomalous low-energy resonance peaks in the magneto-optical conductivity well below the activation gap even at low temperature. The low-energy peaks are enhanced near the insulator-metal phase transition driven by a strong magnetic field. This is a direct consequence of the characteristic Landau level structure and is a dynamical counterpart of quantum oscillations in static quantities, and also shares the same origin (i.e., crossover of the Landau level character). At temperatures sufficiently higher than the activation gap, there are essentially two peaks corresponding to the unhybridized conduction and $f$ electrons, and these peaks develop to form the anomalous peaks at low temperature via the Kondo effect. Our results are qualitatively applicable 
not only to Kondo insulators, but also to other band-inverted insulators such as a quantum well [44-46].

Note added. Recently, we became aware of a recent theoretical work on cyclotron resonance in correlated insulators [49]. We also found experimental reports of cyclotron resonance in InAs/GaSb heterostructures which discuss the effects of the electron-hole hybridization [50-53].

\section{ACKNOWLEDGMENTS}

We thank M. Oshikawa, Y. H. Matsuda, and R. Peters for valuable discussions. This work was supported by Grants-in-Aid for Scientific Research No. JP17K14333 and No. KAKENHI on Innovative Areas "J-Physics" (Grant No. JP18H04318).
[1] D. Shoenberg, Magnetic Oscillations in Metals (Cambridge University Press, Cambridge, 1984).

[2] G. Li, Z. Xiang, F. Yu, T. Asaba, B. Lawson, P. Cai, C. Tinsman, A. Berkley, S. Wolgast, Y. S. Eo, D.-J. Kim, C. Kurdak, J. W. Allen, K. Sun, X. H. Chen, Y. Y. Wang, Z. Fisk, and L. Li, Science 346, 1208 (2014).

[3] B. S. Tan, Y.-T. Hsu, B. Zeng, M. C. Hatnean, N. Harrison, Z. Zhu, M. Hartstein, M. Kiourlappou, A. Srivastava, M. D. Johannes, T. P. Murphy, J.-H. Park, L. Balicas, G. G. Lonzarich, G. Balakrishnan, and S. E. Sebastian, Science 349, 287 (2015).

[4] Z. Xiang, Y. Kasahara, T. Asaba, B. Lawson, C. Tinsman, L. Chen, K. Sugimoto, S. Kawaguchi, Y. Sato, G. Li, S. Yao, Y. L. Chen, F. Iga, J. Singleton, Y. Matsuda, and L. Li, Science 362, 65 (2018).

[5] H. Liu, M. Hartstein, G. J. Wallace, A. J. Davies, M. C. Hatnean, M. D. Johannes, N. Shitsevalova, G. Balakrishnan, and S. E. Sebastian, J. Phys.: Condens. Matter 30, 16LT01 (2018).

[6] J. C. Cooley, C. H. Mielke, W. L. Hults, J. D. Goettee, M. M. Honold, R. M. Modler, A. Lacerda, D. G. Rickel, and J. L. Smith, J. Supercond. 12, 171 (1999).

[7] F. Chen, C. Shang, Z. Jin, D. Zhao, Y. P. Wu, Z. J. Xiang, Z. C. Xia, A. F. Wang, X. G. Luo, T. Wu, and X. H. Chen, Phys. Rev. B 91, 205133 (2015).

[8] M. Shahrokhvand, S. Pezzini, M. R. van Delft, U. Zeitler, N. E. Hussey, and S. Wiedmann, Phys. Rev. B 96, 205125 (2017).

[9] S. Wolgast, Y. S. Eo, K. Sun, Ç Kurdak, F. F. Balakirev, M. Jaime, D.-J. Kim, and Z. Fisk, Phys. Rev. B 95, 245112 (2017).

[10] K. Sugiyama, F. Iga, M. Kasaya, T. Kasuya, and M. Date, J. Phys. Soc. Jpn. 57, 3946 (1988).

[11] T. T. Terashima, A. Ikeda, Y. H. Matsuda, A. Kondo, K. Kindo, and F. Iga, J. Phys. Soc. Jpn. 86, 054710 (2017).

[12] T. T. Terashima, Y. H. Matsuda, Y. Kohama, A. Ikeda, A. Kondo, K. Kindo, and F. Iga, Phys. Rev. Lett. 120, 257206 (2018).

[13] M. Dzero, K. Sun, V. Galitski, and P. Coleman, Phys. Rev. Lett. 104, 106408 (2010).

[14] M. Dzero, J. Xia, V. Galitski, and P. Coleman, Annu. Rev. Condens. Matter Phys. 7, 249 (2016).

[15] J. Knolle and N. R. Cooper, Phys. Rev. Lett. 115, 146401 (2015).

[16] L. Zhang, X.-Y. Song, and F. Wang, Phys. Rev. Lett. 116, 046404 (2016).

[17] H. K. Pal, F. Piéchon, J.-N. Fuchs, M. Goerbig, and G. Montambaux, Phys. Rev. B 94, 125140 (2016).

[18] P. Ram and B. Kumar, Phys. Rev. B 96, 075115 (2017).

[19] H. Shen and L. Fu, Phys. Rev. Lett. 121, 026403 (2018).

[20] S. Grubinskas and L. Fritz, Phys. Rev. B 97, 115202 (2018).

[21] R. Peters, T. Yoshida, and N. Kawakami, Phys. Rev. B 100, 085124 (2019).
[22] O. Erten, P. Ghaemi, and P. Coleman, Phys. Rev. Lett. 116, 046403 (2016).

[23] J. Knolle and N. R. Cooper, Phys. Rev. Lett. 118, 096604 (2017).

[24] I. Sodemann, D. Chowdhury, and T. Senthil, Phys. Rev. B 97, 045152 (2018).

[25] A. H. Castro Neto, F. Guinea, N. M. R. Peres, K. S. Novoselov, and A. K. Geim, Rev. Mod. Phys. 81, 109 (2009).

[26] Y. Ando, J. Phys. Soc. Jpn. 82, 102001 (2013).

[27] N. P. Armitage, E. J. Mele, and A. Vishwanath, Rev. Mod. Phys. 90, 015001 (2018).

[28] M. L. Sadowski, G. Martinez, M. Potemski, C. Berger, and W. A. de Heer, Phys. Rev. Lett. 97, 266405 (2006).

[29] Z. Jiang, E. A. Henriksen, L. C. Tung, Y.-J. Wang, M. E. Schwartz, M. Y. Han, P. Kim, and H. L. Stormer, Phys. Rev. Lett. 98, 197403 (2007).

[30] R. S. Deacon, K.-C. Chuang, R. J. Nicholas, K. S. Novoselov, and A. K. Geim, Phys. Rev. B 76, 081406(R) (2007).

[31] N. Shon and T. Ando, J. Phys. Soc. Jpn. 67, 2421 (1998).

[32] V. P. Gusynin, S. G. Sharapov, and J. P. Carbotte, Phys. Rev. Lett. 98, 157402 (2007).

[33] S. Hill, J. S. Brooks, Z. Q. Mao, and Y. Maeno, Phys. Rev. Lett. 84, 3374 (2000).

[34] M. Kimata, T. Terashima, N. Kurita, H. Satsukawa, A. Harada, K. Kodama, K. Takehana, Y. Imanaka, T. Takamasu, K. Kihou, C.-H. Lee, H. Kito, H. Eisaki, A. Iyo, H. Fukazawa, Y. Kohori, H. Harima, and S. Uji, Phys. Rev. Lett. 107, 166402 (2011).

[35] S. Tonegawa, K. Hashimoto, K. Ikada, Y.-H. Lin, H. Shishido, Y. Haga, T. D. Matsuda, E. Yamamoto, Y. Onuki, H. Ikeda, Y. Matsuda, and T. Shibauchi, Phys. Rev. Lett. 109, 036401 (2012).

[36] G. D. Mahan, Many-Particle Physics, 3rd ed. (Kluwer Academic/Plenum, New York, 2000).

[37] J. D. Denlinger, J. W. Allen, J.-S. Kang, K. Sun, B.-I. Min, D.-J. Kim, and Z. Fisk, $\mathrm{Smb}_{6}$ photoemission: Past and present, in Proceedings of the International Conference on Strongly Correlated Electron Systems, edited by K. Ishida (SCES, Tokyo, Japan, 2013).

[38] M. Okawa, Y. Ishida, M. Takahashi, T. Shimada, F. Iga, T. Takabatake, T. Saitoh, and S. Shin, Phys. Rev. B 92, 161108(R) (2015).

[39] J. Appel and A. W. Overhauser, Phys. Rev. B 18, 758 (1978).

[40] Y. Takada and T. Ando, J. Phys. Soc. Jpn. 44, 905 (1978).

[41] T. Ando, A. B. Fowler, and F. Stern, Rev. Mod. Phys. 54, 437 (1982).

[42] K. Asano and T. Ando, Phys. Rev. B 58, 1485 (1998).

[43] K. Kanki and K. Yamada, J. Phys. Soc. Jpn. 66, 1103 (1997). 
[44] J. Knolle and N. R. Cooper, Phys. Rev. Lett. 118, 176801 (2017).

[45] D. Xiao, C.-X. Liu, N. Samarth, and L.-H. Hu, Phys. Rev. Lett. 122, 186802 (2019).

[46] Z. Han, T. Li, L. Zhang, G. Sullivan, and R.-R. Du, Phys. Rev. Lett. 123, 126803 (2019).

[47] W. T. Fuhrman, J. Leiner, P. Nikolić, G. E. Granroth, M. B. Stone, M. D. Lumsden, L. DeBeer-Schmitt, P. A. Alekseev, J.-M. Mignot, S. M. Koohpayeh, P. Cottingham, W. A. Phelan, L. Schoop, T. M. McQueen, and C. Broholm, Phys. Rev. Lett. 114, 036401 (2015).
[48] C. N. Singh and W.-C. Lee, Phys. Rev. B 97, 241107(R) (2018). [49] P. Rao and I. Sodemann, Phys. Rev. B 100, 155150 (2019).

[50] Y. Vasilyev, S. Suchalkin, K. von Klitzing, B. Meltser, S. Ivanov, and P. Kop'ev, Phys. Rev. B 60, 10636 (1999).

[51] T. P. Marlow, L. J. Cooper, D. D. Arnone, N. K. Patel, D. M. Whittaker, E. H. Linfield, D. A. Ritchie, and M. Pepper, Phys. Rev. Lett. 82, 2362 (1999).

[52] C. Petchsingh, R. J. Nicholas, K. Takashina, N. J. Mason, and J. Zeman, Phys. Rev. B 70, 155306 (2004).

[53] C. Petchsingh, R. J. Nicholas, K. Takashina, and N. J. Mason, Semicond. Sci. Technol. 22, 194 (2007). 